\title{
Limiar anaeróbio e velocidade crítica determinada com diferentes distâncias em nadadores de 10 a 15 anos: relações com a performance e a resposta do lactato sanguíneo em testes de endurance
}

\author{
Camila Coelho Greco ${ }^{1,3}$, Benedito Sérgio Denadai ${ }^{2}$, Ídico Luiz Pellegrinotti ${ }^{1}$, \\ Aline del Bianco Freitas ${ }^{3}$ e Euripedes Gomide ${ }^{3}$
}

\section{RESUMO}

O objetivo deste estudo foi comparar a velocidade crítica $(\mathrm{VC})$ determinada através de diferentes distâncias com o limiar anaeróbio (LAn) e as velocidades máximas mantidas em testes de 20 (V20) e 30 (V30) minutos na natação, verificando se a idade cronológica em jovens nadadores interfere nessas relações. Participaram do estudo 31 nadadores (17 meninas e 14 meninos) divididos segundo a idade cronológica em dois grupos: 10 a 12 anos e 13 a 15 anos. O LAn foi determinado como sendo a velocidade correspondente a $4 \mathrm{mM}$ de lactato sanguíneo. A VC1 (25/50/100m), VC2 $(100 / 200 / 400 m)$ e a VC3 $(50 / 100 / 200 m)$ foram calculadas através do coeficiente angular da reta de regressão linear entre as distâncias e seus respectivos tempos. As V20 e V30 foram determinadas através de três a seis repetições, com coletas de sangue no $10 \mathrm{o}$ minuto e ao final do tiro. Para o grupo de 10 a 12 anos, a VC1 (m/s) $(0,98 \pm 0,17)$ e o LAn $(0,97 \pm 0,12)$ não foram diferentes entre si, sendo maiores do que a VC2 $(0,92 \pm 0,16), \operatorname{VC} 3(0,89 \pm 0,18)$, V20 $(0,92$ $\pm 0,11)$ e V30 $(0,90 \pm 0,11)$. Para o grupo de 13 a 15 anos, a $\mathrm{VC} 1(\mathrm{~m} / \mathrm{s})(1,11 \pm 0,11)$ foi maior do que o LAn $(1,02 \pm$ $0,07)$, V20 $(0,99 \pm 0,09)$, V30 $(0,97 \pm 0,09)$, VC2 $(0,98 \pm$

\footnotetext{
1. Universidade Estadual de Campinas-Unicamp - Campinas, SP.

2. Laboratório de Avaliação da Performance Humana-Unesp - Rio Claro, SP.

3. Centro Universitário Claretiano-CEUCLAR - Batatais, SP.

Recebido em 26/3/02

2 versão recebida em 21/8/02

Aceito em 14/10/02
}

Endereço para correspondência:

Camila Coelho Greco

Rua Marechal Rondon, 599

14020-220 - Ribeirão Preto, SP - Brasil

E-mail: grecocc@hotmail.com
$0,11)$ e VC3 $(1,00 \pm 0,11)$. Pode-se concluir que a distância utilizada na determinação da VC interfere no valor obtido, independente da idade cronológica. A VC determinada com distâncias entre 50 e $400 \mathrm{~m}$ pode ser utilizada na avaliação da capacidade aeróbia de crianças e adolescentes, substituindo os testes contínuos máximos com durações próximas a 20 ou 30 minutos.

Palavras-chave: Limiar anaeróbio. Velocidade crítica. Lactato. Crianças. Natação.

\section{ABSTRACT}

Anaerobic threshold and critical speed determined with different distances in swimmers aged 10 to 15 years: relationship with the performance and blood lactate response during endurance tests

The objective of this study was to compare the critical speed (CS) determined from different distances with the anaerobic threshold (AnT) and the maximum speeds in tests of 20 (S20) and 30 (S30) minutes, and to check if the chronological age of young swimmers can influence these relations. 31 swimmers (17 girls and 14 boys) participated in this study, divided by chronological age into two groups: 10-12 and 13-15 years. The AnT was determined as the velocity corresponding to $4 \mathrm{mM}$ of blood lactate. The CSI (25/50/100 m), CS2 (100/200/400 m), and the CS3 (50/100/ $200 \mathrm{~m}$ ) had been calculated through the slope of the linear regression between the distances and their respective times. The S2O and S30 were determined through 3 to 6 trials, with blood collections at the $10^{\text {th }} \mathrm{min}$ and at the end of each test. For the 10-12 group, CS1 (m/s) $(0.98 \pm 0.17)$ and AnT $(0.97 \pm 0.12)$ were not different, being higher than CS2 $(0.92$ $\pm 0.16), \operatorname{CS} 3(0.89 \pm 0.18), S 20(0.92 \pm 0.11)$ and $S 30(0.90$ $\pm 0.11)$. For the $13-15$ group, CS1 $(\mathrm{m} / \mathrm{s})(1.11 \pm 0.11)$ was higher than AnT $(1.02+0.07)$, S20 (0.99 + 0.09), S30 (0.97 $\pm 0.09), \operatorname{CS2}(0.98 \pm 0.11)$ and CS3 $(1.00 \pm 0.11)$. It can be 
concluded that the distance used for the determination of CS interferes on its value, irrespective of the chronological age. CS determined with distances between 50 and $400 \mathrm{~m}$ can be used for evaluation of the aerobic capacity of children and adolescents, substituting the maximum continuous tests of 20 or 30 minutes.

Key words: Anaerobic threshold. Critical speed. Lactate. Children. Swimming.

\section{INTRODUÇÃO}

Crianças e adolescentes apresentam menores valores de lactato sanguíneo em resposta ao exercício (submáximo e máximo) quando comparado com adultos ${ }^{1}$. Estas diferenças são atribuídas às menores concentrações de enzimas glicolíticas como a fosfofrutoquinase (PFK) e maiores concentrações de enzimas aeróbias como a succinato desidrogenase $(\mathrm{SDH})^{2}$. As crianças também apresentam menores concentrações de hormônios esteróides como a testosterona, que com o processo de maturação tem seus níveis elevados, aumentando a massa muscular e a capacidade anaeróbia $^{3,4}$. Essas modificações determinam um deslocamento à esquerda da curva lactato $\mathrm{x}$ intensidade de esforço, reduzindo o limiar de lactato (LL) (ponto de inflexão na curva lactato e intensidade) com o processo de maturaçãa $0^{5,6}$.

Em adultos, Heck et al. ${ }^{7}$ propuseram a identificação da máxima fase estável de lactato sanguíneo (MLACSS), que pode ser definida como a maior intensidade de exercício de carga constante em que ainda existe equilíbrio entre a taxa de liberação e remoção de lactato no sangue, empregando um valor fixo de $4 \mathrm{mM}$. Esse critério pode ser interessante, pois não exige a realização de quatro a seis sessões de exercícios submáximos, com carga constante e em dias separados, para a determinação da MLACSS. Alguns estudos, entretanto, têm demonstrado que a concentração de lactato correspondente à MLACSS pode variar entre os indivíduos ${ }^{8}$ e depender do tipo de exercício realizado . $^{9}$

Para a determinação da MLACSS durante a corrida em crianças e adolescentes, Williams e Armstrong ${ }^{10}$ sugerem um valor fixo de $2,5 \mathrm{mM}$ de lactato sanguíneo. Entretanto, em alguns estudos realizados na corrida em meninos com idade de 11 anos ${ }^{11,12}$ ou em meninos e meninas de 12 anos $^{13}$, foram verificadas concentrações próximas a $5 \mathrm{mM}$ e $3,9 \mathrm{mM}$, respectivamente, como sendo correspondentes à MLACSS. Beneke et al. ${ }^{14}$ encontraram em adolescentes (14,3 anos) durante o exercício na bicicleta um valor de $4,5 \mathrm{mM}$ como correspondente à MLACSS. Beneke et al. ${ }^{14}$ apontam que as diferenças entre seus resultados e os dos estudos citados anteriormente ${ }^{10-12}$ ocorreram em função do tipo de protocolo empregado na determinação da MLACSS. Enquanto Williams e Armstrong ${ }^{10}$ e Mocellin et al. ${ }^{11,12}$ determinaram as concentrações de lactato em protocolos de carga constante relativamente curtos (10 e 16 minutos, respectivamente), Beneke et al. ${ }^{14}$ utilizaram um protocolo com 20 minutos. Estudos realizados em adultos apontam que são necessários 30 minutos de carga constante para a determinação da cinética de lactato e, conseqüentemente, da MLACSS $^{15,16}$. Como a concentração de MLACSS parece depender também do tipo de exercício ${ }^{9}$, não é possível atribuir exclusivamente ao protocolo as diferenças entre os estudos citados anteriormente. Deve-se ressaltar ainda que não foram encontrados estudos que analisaram a MLACSS na natação em crianças e adolescentes.

Entre os diferentes métodos não-invasivos utilizados para predizer a resposta do lactato sanguíneo durante o exercício, a potência crítica (PC) (para a bicicleta ergométrica ou ergômetro de braço) e a velocidade crítica (corrida e natação) têm apresentado elevados índices de correlação com a intensidade correspondente a $4 \mathrm{mM}$ de lactato sanguíneo e com a performance aeróbia ${ }^{17-19}$. Teoricamente, a potência/velocidade crítica é definida como sendo a mais alta intensidade de exercício que pode ser mantida por longo período de tempo sem exaustão ${ }^{20}$. Seu conceito é baseado na relação hiperbólica entre intensidades predeterminadas e seus respectivos tempos de exaustão. Especificamente para a natação e a corrida, a velocidade crítica (VC) pode ser obtida pela regressão linear entre distâncias fixas e seus respectivos tempos, sendo que a VC corresponde ao coeficiente angular da reta obtida ${ }^{18,19}$.

A determinação da potência crítica pode ser protocolodependente. Alguns estudos têm verificado que a escolha das cargas empregadas na sua predição pode determinar diferentes valores de potência crítica ${ }^{21,22}$. Para a VC, essa influência da seleção das cargas (distâncias) ainda não foi apropriadamente estudada. Esse aspecto pode ser particularmente importante quando se utiliza a VC para a prescrição da intensidade do treinamento ${ }^{23}$.

A VC apresenta muitas vantagens, como a facilidade de aplicação e análise de grande número de atletas, pode ser realizada durante as sessões de treino e, principalmente, sem a necessidade da utilização de equipamentos caros ou coleta de sangue ${ }^{23}$. Esses fatores devem ser levados em consideração quando a população avaliada é de crianças e adolescentes. Entretanto, poucos foram os estudos que verificaram a validade da VC para a determinação da resposta de lactato sanguíneo em crianças e adolescentes ${ }^{23-25}$, principalmente durante a realização de exercícios submáximos de carga constante. Com isso, os objetivos deste estudo foram: a) comparar a $\mathrm{VC}$ determinada através de diferentes distâncias com o limiar anaeróbio (LAn) durante a natação; b) comparar a VC e o LAn com as velocidades máximas mantidas em testes de 20 e 30 minutos; c) analisar a res- 
posta do lactato durante os testes de endurance de 20 e 30 minutos e; d) verificar se a idade cronológica em nadadores de 10 a 15 anos interfere nas relações citadas anteriormente.

\section{MATERIAL E MÉTODOS}

\section{Sujeitos}

Participaram do estudo 31 nadadores (17 meninas e 14 meninos) divididos de acordo com a idade cronológica em dois grupos:

1) 10 a 12 anos: 15 nadadores (seis meninos e nove meninas) que objetivavam principalmente a melhora dos estilos e que tinham pelo menos um a dois anos de experiência na modalidade. Suas características físicas eram (média \pm DP): idade $=11,1 \pm 0,7$ anos, massa corporal $=41,4 \pm 8,0 \mathrm{~kg}$ e estatura $=146,4 \pm 8,0 \mathrm{~cm}$. Os nadadores estavam envolvidos em um programa de treinamento cinco vezes por semana com um volume médio semanal entre 5.500 e $9.500 \mathrm{~m}$.

2) 13 a 15 anos: 16 nadadores (oito meninos e oito meninas) que estavam na fase de treinamento e que tinham pelo menos três a cinco anos de experiência na modalidade. Suas características físicas eram: idade $=14,1 \pm 0,9$ anos, massa corporal $=53,8 \pm 5,5 \mathrm{~kg}$ e estatura $=159,8 \pm$ $6,0 \mathrm{~cm}$. Os nadadores estavam envolvidos em um programa de treinamento cinco vezes por semana com um volume médio semanal entre 13.500 e $17.500 \mathrm{~m}$.

Antes da participação nos protocolos, os pais ou responsáveis e os indivíduos foram informados de todos os procedimentos inerentes aos testes, assinando um termo de consentimento concordando com a participação no estudo. O estudo foi aprovado pelo Comitê de Ética em Pesquisa da Unicamp.

\section{Determinação do limiar anaeróbio}

O LAn foi determinado através da metodologia proposta por Mader et al. ${ }^{26}$, utilizando-se uma concentração fixa de $4 \mathrm{mM}$ de lactato sanguíneo. Foram realizadas duas repetições submáximas de $200 \mathrm{~m}$, a 90 e $95 \%$ da velocidade máxima para a distância, que foram separadas entre si por 15 a 20 minutos de recuperação passiva. Após um, três e cinco minutos de cada repetição foram coletados $25 \mu \mathrm{lde}$ sangue do lóbulo da orelha, através de um capilar heparinizado. O sangue foi imediatamente transferido para microtúbulos de polietileno com tampa tipo Eppendorff de $1,5 \mathrm{ml}$, contendo $50 \mu \mathrm{l}$ de fluoreto de sódio $(\mathrm{NaF})$ a $1 \%$ e este foi armazenado em gelo. A análise do lactato foi realizada através do analisador eletroquímico modelo YSL 1500 Sport. A velocidade correspondente a $4 \mathrm{mM}$ foi determinada por interpolação linear, entre a mais alta concentração de lactato de cada tiro e suas respectivas velocidades.

\section{Determinação da velocidade crítica}

Para a determinação da VC, foram realizadas performances máximas nas distâncias de 25, 50, 100, 200 e 400m na piscina, anotando-se os respectivos tempos. Os tiros foram realizados em ordem aleatória durante as sessões de treinamento, sendo uma tentativa por sessão. A VC foi determinada através do coeficiente angular (b) da reta de regressão linear entre as distâncias e os respectivos tempos obtidos em cada repetição. Para a determinação da VC1 foram utilizadas as distâncias de 25,50 e $100 \mathrm{~m}$; para a VC2 foram utilizadas as distâncias de 100, 200 e 400m; e para a VC3 foram utilizadas as distâncias de 50, 100 e 200m. Estudos anteriores verificaram a validade da determinação da VC em crianças e adolescentes dentro do modelo utilizado neste estudo ${ }^{23-25}$.

\section{Determinação das velocidades de 20 e 30 minutos}

O objetivo deste teste foi determinar as maiores velocidades que podiam ser mantidas por 20 (V20) e 30 minutos (V30). Dos 31 nadadores que participaram do estudo, 23 iniciaram a realização desses testes. Destes, em apenas 12 foi possível determinar perfeitamente a V20 e V30. Isso ocorreu basicamente em função do elevado número de sessões experimentais (10 a 12) que cada nadador precisou realizar em todo o estudo.

As V20 e V30 foram determinadas através de três a seis repetições em velocidades constantes controladas a cada $25 \mathrm{~m}$. A primeira repetição foi realizada a $85 \%$ da velocidade dos 400m, com todos os nadadores conseguindo completar os 30 minutos. Posteriormente, acresceu-se de 1 a $2 \%$ na intensidade entre cada tentativa, até que fossem obtidas as maiores velocidades constantes que permitiam durações de aproximadamente 20 e 30 minutos. Adotou-se como critério para a interrupção do teste quando o nadador entrou em exaustão voluntária, ou quando não conseguiu manter o tempo previsto a cada 25 metros (aumento maior que dois segundos) por três vezes consecutivas, embora tenha sido estimulado verbal e visualmente para a correção da velocidade. No 10 o minuto e ao final de cada repetição, foram coletados $25 \mu \mathrm{l}$ de sangue arterializado, através de um capilar heparinizado, para posterior análise do lactato sanguíneo.

\section{Análise estatística}

A comparação dos valores de VC, LAn, V20, V30 e o lactato durante V20 e V30 foi feita através da ANOVA para dois caminhos, complementada pelo teste de Scheffé. A correlação entre as variáveis VC, LAn, V20 e V30 foi realizada através do teste de correlação de Pearson. O nível de significância foi de $p \leq 0,05$. 
TABELA 1

Valores médios \pm DP das velocidades $(\mathrm{m} / \mathrm{s})$ correspondentes ao limiar anaeróbio (4mM) (LAn) e as velocidades críticas (VC) 1, VC2 e VC3 para os grupos de 10 a 12 e 13 a 15 anos

\begin{tabular}{lcccc}
\hline & LAn & $\begin{array}{c}\text { VC1 } \\
(\mathbf{2 5 / 5 0 / 1 0 0 m})\end{array}$ & $\begin{array}{c}\text { VC2 } \\
(\mathbf{1 0 0 / 2 0 0 / 4 0 0 m})\end{array}$ & $\begin{array}{c}\text { VC3 } \\
(\mathbf{5 0 / 1 0 0 / 2 0 0 m})\end{array}$ \\
& & & & \\
$10-12$ anos $(\mathrm{N}=15)$ & $0,90 \pm 0,13$ & $0,92 \pm 0,16$ & $0,85 \pm 0,16^{* \dagger}$ & $0,85 \pm 0,15^{* \dagger}$ \\
$13-15$ anos $(\mathrm{N}=16)$ & $1,04 \pm 0,11^{\dagger}$ & $1,16 \pm 0,15$ & $0,99 \pm 0,12^{\dagger}$ & $1,02 \pm 0,14^{\dagger}$ \\
\hline$* \mathrm{p}<0,05$ em relação ao LAn dentro do mesmo grupo. & & \\
$+\mathrm{p}<0,05$ em relação à VC1 dentro do mesmo grupo. & & \\
\hline
\end{tabular}

\section{RESULTADOS}

A tabela 1 apresenta os valores médios \pm DP das velocidades correspondentes ao LAn $(4 \mathrm{mM})$ e as velocidades críticas (VC) 1 (25/50/100), $2(100 / 200 / 400)$ e $3(50 / 100 / 200)$ determinadas nos 31 nadadores, para os grupos de 10 a 12 e 13 a 15 anos. No grupo de 10 a 12 anos, o LAn e a VC1 foram significantemente maiores do que a VC2 e VC3. Não houve diferença entre o LAn e a VC1. No grupo de 13 a 15 anos, VC1 foi significantemente maior do que o LAn, VC2 e VC3. Não houve diferença entre o LAn, a VC2 e VC3.

As correlações entre as VC1 $(0,95)$, VC2 $(0,93)$ e VC3 $(0,94)$ com o LAn para o grupo de 10 a 12 anos foram significantes. Do mesmo modo, foram significantes as correlações entre VC1 $(0,82)$, VC2 $(0,69)$ e VC3 $(0,93)$ com o LAn para o grupo de 13 a 15 anos.

A tabela 2 apresenta os valores médios \pm DP da VC1 (25/ 50/100), 2 (100/200/400) e $3(50 / 100 / 200)$, do LAn, V20 e V30 determinadas em 12 nadadores, para os grupos de 10 a 12 e 13 a 15 anos. Para o grupo de 10 a 12 anos, a VC1 e o

TABELA 2

Valores médios $\pm \mathrm{DP}(\mathrm{m} / \mathrm{s})$ da velocidade crítica

(VC) 1, VC2 e VC3, do limiar anaeróbio (4mM)

(LAn) e das velocidades de 20 (V20) e 30 minutos

(V30), para os grupos de 10 a 12 e 13 a 15 anos

\begin{tabular}{|c|c|c|}
\hline & $\begin{array}{c}10-12 \text { anos } \\
N=6\end{array}$ & $\begin{array}{c}13-15 \text { anos } \\
N=6\end{array}$ \\
\hline VC1 (25/50/100m) & $0,98 \pm 0,17$ & $1,11 \pm 0,11$ \\
\hline VC2 (100/200/400m) & $0,92 \pm 0,16^{* \dagger}$ & $0,98 \pm 0,11^{*}$ \\
\hline VC3 (50/100/200m) & $0,89 \pm 0,18^{* \dagger}$ & $1,00 \pm 0,11^{*}$ \\
\hline LAn & $0,97 \pm 0,12$ & $1,02 \pm 0,07^{*}$ \\
\hline V20 & $0,92 \pm 0,11^{*+}$ & $0,99 \pm 0,09 *$ \\
\hline V30 & $0,90 \pm 0,11^{*++}$ & $0,97 \pm 0,09 *+$ \\
\hline
\end{tabular}

* $\mathrm{p}<0,05$ em relação à VC1 dentro do mesmo grupo.

$\dagger \mathrm{p}<0,05$ em relação ao LAn dentro do mesmo grupo.

$+\mathrm{p}<0,05$ em relação à V20 dentro do mesmo grupo.
LAn não foram diferentes entre si, sendo significantemente maiores do que a VC2, VC3, V20 e V30. Não houve diferença entre a VC2 e VC3 em relação à V20 e à V30. A V20 foi significantemente maior do que a V30. Para o grupo de 13 a 15 anos, a VC1 foi maior do que o LAn, V20, V30, VC2 e VC3. A VC2 e VC3 foram iguais ao LAn, a V20 e a V30. A V20 foi significantemente maior do que a V30.

Os valores de correlação para o grupo de 10 a 12 anos entre as VC e o LAn foram elevados $(0,98$ a 0,99$)$. Do mesmo modo, as correlações das VC com a V20 e a V30 (0,84 a $0,96)$ e entre o LAn e a V20 $(0,93)$ e a V30 $(0,91)$ foram significantes. Para o grupo de 13 a 15 anos, as correlações entre as VC e o LAn $(0,85$ a 0,91$)$, das VC com a V20 e a V30 $(0,88$ a 0,94$)$ e entre o LAn e a V20 $(0,93)$ e a V30 $(0,93)$ também foram significantes.

A figura 1 apresenta os valores individuais das concentrações de lactato sanguíneo $(\mathrm{mM})$ obtidas nos testes máximos de 20 e 30 minutos, para o grupo de 10-12 anos. Os valores médios \pm DP das concentrações $(\mathrm{mM})$ obtidas no teste de 20 minutos foram de 2,14 $\pm 0,7$ e 2,09 $\pm 0,7$, respectivamente no $10^{\circ}$ minuto e ao final do teste. No teste de 30 minutos os valores obtidos foram de 1,77 $\pm 0,5$ e 1,77 \pm 0,5 , respectivamente. Não houve diferença significante entre os dois momentos da coleta (10 minuto x final do

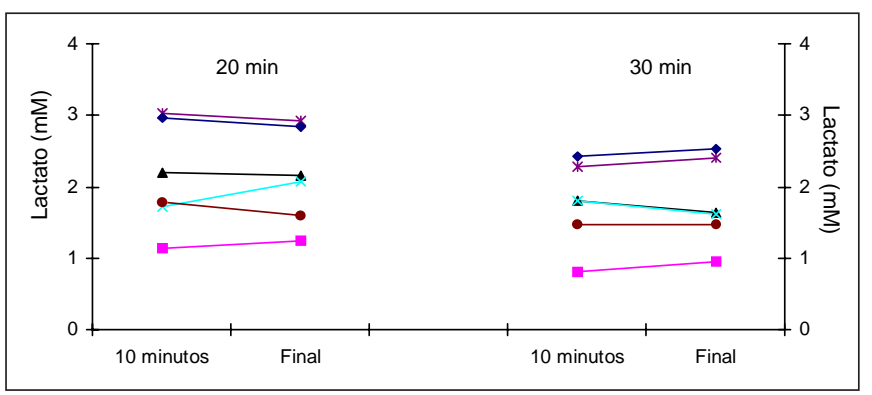

Fig. 1 - Valores individuais das concentrações de lactato sanguíneo obtidas no $10^{\circ}$ min e ao final dos testes máximos de 20 e $30 \mathrm{~min}$, para o grupo de 10 a 12 anos 


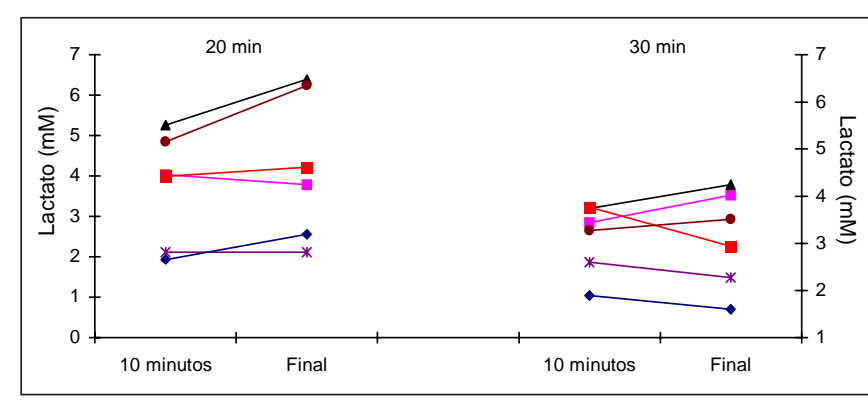

Fig. 2 - Valores individuais das concentrações de lactato sanguíneo obtidas no $10^{\circ}$ min e ao final dos testes máximos de 20 e 30 min, para o grupo de 13 a 15 anos

teste) quando comparados dentro do mesmo teste (V20 e V30). Entretanto, os dois valores obtidos no teste de 20 minutos foram maiores do que os de 30 minutos.

A figura 2 apresenta os valores individuais das concentrações de lactato sanguíneo $(\mathrm{mM})$ obtidas nos testes máximos de 20 e 30 minutos, para o grupo de 13 a 15 anos. Os valores médios \pm DP das concentrações $(\mathrm{mM})$ obtidas no teste de 20 minutos foram de 3,81 $\pm 1,22$ e 4,32 $\pm 1,69$, respectivamente no $10^{\circ}$ minuto e ao final do teste. No teste de 30 minutos os valores obtidos foram de 2,61 $\pm 0,61 \mathrm{e}$ $2,56 \pm 1,01$, respectivamente. Não houve diferença significante entre os dois momentos da coleta $\left(10^{\circ}\right.$ minuto $\mathrm{x}$ final do teste) no teste de 30 minutos. Entretanto, no teste de 20 minutos o valor obtido no $10^{\circ}$ minuto foi menor do que o medido no final do teste. Os valores obtidos no teste de 20 minutos foram maiores do que os de 30 minutos.

\section{DISCUSSÃO}

O objetivo central deste estudo foi comparar a VC determinada a partir de diferentes distâncias com o LAn e as velocidades máximas mantidas em testes de 20 e 30 minutos durante a natação, verificando se a idade cronológica de nadadores de 10 a 15 anos interfere nas relações citadas anteriormente. Os principais achados deste estudo foram: a) a combinação de diferentes distâncias interfere nos valores de VC, sem existir, entretanto, influência da idade cronológica; b) a relação entre a VC determinada a partir da combinação de diferentes distâncias com o LAn (4mM) é influenciada pela idade cronológica, com o valor fixo de 4mM para o grupo de 10-12 anos, determinando intensidade maior do que a que pode ser mantida por 20 ou $30 \mathrm{mi}-$ nutos; e c) a VC2 e VC3 podem prever a maior velocidade que pode ser mantida por 20 ou 30min, independente da idade cronológica.

Bishop et al. ${ }^{21}$ verificaram que a combinação de cargas que permitiam tempo de exaustão entre 68 e 193 segundos determinou potência crítica na bicicleta ergométrica maior do que quando foram utilizadas cargas em que o tempo de exaustão ficou entre 193 e 485 segundos (201 e 164W, respectivamente). Resultados semelhantes foram obtidos no ergômetro de braço por Calis e Denadai ${ }^{22}$. Bishop et al. ${ }^{21}$ propõem que a influência da seleção das cargas na determinação da potência crítica ocorre principalmente quando o tempo de exaustão permitido pela carga é inferior a três minutos. Esse fenômeno ocorreria em função da "inércia aeróbia", pois no início do exercício o consumo de oxigênio aumenta de forma monoexponencial até atingir a fase estável após dois-três minutos. Se essa fase não é considerada, a relação entre a potência e o tempo é modificada, resultando em maior $\mathrm{PC}^{27}$. Esse comportamento parece estar presente também na determinação da VC, em que são empregadas distâncias fixas e não o tempo de exaustão. No presente estudo, a combinação das distâncias menores (25/50/100m), portanto, com os menores tempos de execução (entre 14 e 121 segundos), determinou os maiores valores de VC para os dois grupos analisados (10-12 anos e 13-15 anos), mostrando que a idade cronológica não influencia nessa relação.

Wakayoshi et al. ${ }^{28}$ foram os primeiros a estender o conceito de potência crítica para a natação, determinando neste caso a VC. Nesse estudo, realizado em um swimmingflume, os autores não observaram diferença $(1,16 \pm 0,05$ vs. $1,16 \pm 0,03 \mathrm{~m} / \mathrm{s})$ e alta correlação $(\mathrm{r}=0,94, \mathrm{p}<0,01)$ entre a VC e o LAn em um grupo de nadadores adultos (1624 anos) e bem treinados, sugerindo que a VC pode ser utilizada para predizer o LAn. Posteriormente, Wakayoshi et al. ${ }^{29}$ também encontraram alta correlação $(0,91, \mathrm{p}<0,01)$ entre a VC e o LAn, verificando ainda que a VC correspondeu à MLACSS em um grupo de nadadores treinados colegiais (18-20 anos). Nesse estudo a VC foi determinada na piscina com distâncias fixas (200/400m) e não com o tempo de exaustão (velocidade fixa).

Diferentemente desses resultados, verificamos em nosso estudo, no grupo de 10-12 anos, que somente a VC1 (0,92 $\pm 0,16 \mathrm{~m} / \mathrm{s})$ foi semelhante ao LAn $(0,90 \pm 0,13 \mathrm{~m} / \mathrm{s})$, a VC2 $(0,85 \pm 0,16 \mathrm{~m} / \mathrm{s})$ e a VC3 $(0,85 \pm 0,15 \mathrm{~m} / \mathrm{s})$ sendo menores do que as velocidades citadas anteriormente. Esse resultado, entretanto, está de acordo com o encontrado recentemente por Denadai et al. ${ }^{25} \mathrm{em}$ um grupo de nadadores da mesma faixa etária (10-12 anos). Denadai et al. ${ }^{25}$, utilizando distâncias $(50 / 100 / 200 \mathrm{~m})$ semelhantes às empregadas por Wakayoshi et al. ${ }^{19,29}$, encontraram que a VC foi significantemente menor do que o LAn, independentemente do nível de treinamento das crianças na natação.

A resposta do lactato sanguíneo ao exercício (submáximo e máximo) em crianças pode explicar, pelo menos em parte, esses resultados. Eriksson e Saltin ${ }^{30}$ e Berg et al. ${ }^{31}$ demonstraram em indivíduos de seis a 17 anos que a ativi- 
dade das enzimas glicolíticas tende a aumentar, enquanto a das enzimas oxidativas a diminuir com o avanço da idade. Isso sugere que existe menor proporção entre a atividade enzimática anaeróbia-aeróbia em crianças, a qual pode estar relacionada com a redução na produção de lactato, aumento da oxidação do lactato, ou ambos. Diversos estudos têm verificado que a resposta de lactato em crianças expressa por diversas terminologias e critérios (limiar de lactato, limiar anaeróbio) diminui com o avanço da idade cronológica (10 aos 18 anos), tanto expressa em valores relativos ao consumo máximo de oxigênio ${ }^{1,32}$ ou na velocidade de corrida ${ }^{6,33}$.

Confirmando as possibilidades apontadas anteriormente, verificamos no grupo de 13-15 anos do nosso estudo que a $\operatorname{VC} 2(\mathrm{~m} / \mathrm{s})(0,99 \pm 0,12 \mathrm{~m} / \mathrm{s}), \operatorname{VC} 3(1,02 \pm 0,14 \mathrm{~m} / \mathrm{s})$ e o LAn $(1,04 \pm 0,11 \mathrm{~m} / \mathrm{s})$ não foram significantemente diferentes e menores do que a $\mathrm{VC} 1(1,16 \pm 0,15 \mathrm{~m} / \mathrm{s})$. Assim, com o início do processo de maturação, os adolescentes já apresentam resultados semelhantes ( $\mathrm{VC}=\mathrm{LAn})$ aos encontrados em nadadores adultos, quando as determinações da VC são feitas com distâncias semelhantes $(50-400 \mathrm{~m})$ às do nosso estudo ${ }^{19,29}$.

A concentração de lactato correspondente à MLACSS em crianças e adolescentes tem produzido resultados conflitantes. Enquanto Williams e Armstrong ${ }^{10}$ sugerem para a corrida um valor fixo de 2,5mM, Mocellin et al. ${ }^{11,12}$ também durante a corrida e Beneke et al. ${ }^{14}$ no ciclismo verificaram concentrações próximas a $5 \mathrm{mM}$ e $4,5 \mathrm{mM}$ como correspondentes à MLACSS, respectivamente. Essas discordâncias provavelmente podem ser explicadas pelo tipo de exercício ${ }^{9}$, protocolos empregados para a determinação da MLACSS $^{10-12,14} \mathrm{e}$, como visto em nosso estudo, pela faixa etária analisada.

Em nosso estudo as concentrações de lactato $(\mathrm{mM})$ no grupo de 10 a 12 anos nos tiros máximos de 20 (10ํㅡำ to $=2,14 \pm 0,7$; final do teste $=2,09 \pm 0,7)$ e 30 minutos $(10$ o minuto $=1,77 \pm 0,5$; final do teste $=1,77 \pm 0,5)$ foram menores do que os valores obtidos no grupo de 13 a 15 anos para os testes de 20 (10 minuto $=3,81 \pm 1,22$; final do teste $=4,32 \pm 1,69)$ e 30 minutos $\left(10^{\circ}\right.$ minuto $=2,61 \pm$ $0,61$; final do teste $=2,56 \pm 1,01)$, mostrando que a idade cronológica interfere na resposta de lactato sanguíneo em testes de endurance com velocidades constantes.

Essa influência da idade cronológica e os valores médios de lactato, principalmente para a V20, confirmam e validam os resultados que foram observados nos dois grupos quando a velocidade de LAn é comparada com as VC $(1,2$ e 3$)$ e a V20 e V30. Assim, para o grupo de 10 a 12 anos, a velocidade de LAn com concentração fixa de $4 \mathrm{mM}$ não parece ser adequada quando o objetivo é a prescrição de intensidade de nado que possa ser realizada por $20 \mathrm{ou}$ 30min, ou para a prescrição do treino intervalado de características aeróbias. Entretanto, com o avançar da idade cronológica (13 a 15 anos), provavelmente em função do processo de maturação, a velocidade de $4 \mathrm{mM}$ (LAn) já começa a ser um índice mais apropriado para a seleção de cargas para treinos de endurance (contínuos ou intervalados). Entretanto, os valores médios de lactato encontrados em nosso estudo devem ser analisados com restrições, em função da variabilidade interindividual relativamente grande e também do número reduzido $(n=6)$ de participantes em cada grupo. Embora os dados mostrem claramente uma diferença entre os grupos, ainda não nos parece adequado fixar esses valores médios como referência para a determinação da intensidade de exercício, principalmente no grupo de 10-12 anos, como freqüentemente feito em adultos $(4 \mathrm{mM})^{7}$.

Essas mesmas restrições devem, em princípio, ser feitas para a análise das relações entre o LAn, VC, V20 e V30, em razão do número relativamente reduzido de nadadores em cada grupo. Deve-se ressaltar, entretanto, que as relações entre as $\mathrm{VC}$ e o LAn foram exatamente iguais quando se analisaram os 31 nadadores ou quando a amostra ficou restrita aos 12 que fizeram todos os testes.

Independente desses aspectos, a $\mathrm{VC} 2 \mathrm{e} \mathrm{VC} 3$, foram bastante semelhantes à V20 e V30, o que na natação pode ser interessante. É bastante comum o emprego pelos técnicos de testes de 20 ou 30 minutos (sem coleta de sangue) para avaliação aeróbia dos seus atletas, prescrevendo inclusive intensidades de treinamento em percentuais da velocidade encontrada nesses testes ${ }^{34,35}$. Esses testes, entretanto, ao serem aplicados em nadadores mais novos e/ou menos experientes, podem ter sua validade diminuída, em função da motivação e da própria experiência em testes mais longos, podendo comprometer os valores obtidos. Já com o emprego da VC2 e VC3, esses aspectos podem ser minimizados, pois as distâncias nadadas são bem menores (até $400 \mathrm{~m})$. Deve-se salientar que a relação entre a VC2 e VC3 com a V20 e V30 não se mostrou dependente da idade cronológica, enquanto a intensidade correspondente a $4 \mathrm{mM}$ superestimou a V20 e V30 no grupo de 10 a 12 anos. Dados bem recentes de Dekerle et al. ${ }^{36}$ mostraram que a VC determinada com as distâncias de 200 e 400m em nadadores adultos é bastante semelhante à V30, confirmando os resultados do nosso estudo de que a predição da V30 pela VC (com as distâncias adequadas) é realmente independente da idade cronológica.

Com base nesses resultados podemos concluir que a distância utilizada na determinação da VC interfere no valor obtido independente da idade cronológica, podendo provocar diferentes adaptações quando utilizada para a prescrição do treinamento. Por outro lado, a relação entre a VC 
e o LAn (4mM), é dependente da distância empregada na determinação da VC e da idade cronológica. A VC determinada com distâncias entre 50 e 400m pode ser utilizada na avaliação da capacidade aeróbia de crianças e adolescentes, substituindo com vantagens os testes contínuos máximos com durações próximas a 20 ou $30 \mathrm{~min}$. A resposta de lactato em crianças de 10 a 12 anos em testes máximos de 20 ou 30min é diferente de adolescentes (13 a 15 anos), com o LAn $(4 \mathrm{mM})$ representando intensidade que não pode ser mantida por esse tempo.

Todos os autores declararam não haver qualquer potencial conflito de interesses referente a este artigo.

\section{REFERÊNCIAS}

1. Armstrong N, Welsman JR. Assessment and interpretation of aerobic fitness in children and adolescents. Exerc Sport Sci Rev 1994;22:43576.

2. Eriksson BO, Gollnick PD, Saltin B. Muscle metabolism and enzyme activities after training in boys 11-13 years old. Acta Paediatr Scand 1973;87:485-97.

3. Gutman E, Hanzlikova V, Lojdaz Z. Effect of androgen on histochemical fiber type. Histochemie 1973;24:287-91

4. Krotkiewski M, Kral JG, Karlsson J. Effects of castration and testosterone substitution on body composition and muscle metabolism in rats. Acta Physiol Scand 1980;109:233-7.

5. Sjodin B, Jacobs I, Karlsson J. Onset of blood lactate accumulation and enzyme activities in M. Vastus Lateralis in man. Int J Sports Med 1981; 2:166-70.

6. Tanaka H. Predicting running velocity at blood lactate threshold from running performance tests in adolescents boys. Eur J Appl Physiol 1986; 55:344-8.

7. Heck H, Mader A, Hess G, Mucke S, Muller R, Hollmann W. Justification of the 4mmol/L lactate threshold. Int J Sports Med 1985;6:117-30.

8. Stegmann H, Kindermann W, Schnabel A. Lactate kinetics and individual anaerobic threshold. Int J Sports Med 1981;2:160-5.

9. Beneke R, Von Duvillard SP. Determination of maximal lactate steady state response in selected sports events. Med Sci Sports Exerc 1996;28: 241-6.

10. Williams JR, Armstrong N. Relationship of maximal lactate steady state to performance at fixed blood lactate reference values in children. Pediatr Exerc Sci 1991;3:333-41.

11. Mocellin R, Heusgen M, Korsten-Reck O. Maximal lactate steady state blood lactate levels in 11-year-old boys. Eur J Pediatr 1990;149:771-3.

12. Mocellin R, Heusgen M, Gildein HP. Anaerobic threshold and maximal lactate steady-state in prepubertal boys. Eur J Appl Physiol 1991;62:5660 .

13. Billat V, Gratas-Delamarche A, Monnier M, Delamarche P. A test to approach maximal lactate steady-state in 12-year-old boys and girls. Arch Physiol Biochem 1995;103:65-72.

14. Beneke R, Schwarz V, Leithauser R, Hutler M, Von Duvillard SP. Maximal lactate steady state in children. Pediatr Exerc Sci 1996;8:328-36.

15. Aunola S, Rusko H. Does anaerobic threshold correlate with maximal lactate steady sate? J Sports Sci 1992;10:309-23.
16. Mognoni P, Sirtori MD, Lorenzelli F, Cerretelli P. Physiological response during prolonged exercise at the power output corresponding to the blood lactate threshold. Eur J Appl Physiol 1990;60:239-43.

17. Housh TJ, de Vries HA, Tichy MW, Smyth KD. The relationship between critical power and the onset of blood lactate accumulation. J Sports Med Phys Fitness 1991;31:31-6.

18. Kranenburg KJ, Smith DJ. Comparison of critical speed determined from track running and treadmill tests in elite runners. Med Sci Sports Exerc 1996;28:614-8.

19. Wakayoshi K, Yoshida T, Udo M, Kasai T, Moritani T, Mutoh Y, et al. A simple method for determining critical speed as swimming fatigue threshold in competitive swimming. Int J Sports Med 1992;13:367-71.

20. Monod H, Scherrer J. The work capacity of a synergic muscular group. Ergonomics 1965;8:329-38.

21. Bishop D, Jenkins DG, Howard A. The critical power is dependent on the duration of the predictive exercise tests chosen. Int J Sports Med 1998;19:125-9.

22. Calis JFF, Denadai BS. Influência das cargas selecionadas na determinação da potência crítica determinada no ergômetro de braço em dois modelos lineares. Rev Bras Med Esporte 2000;6:1-4.

23. Hill DW, Steward RPJR, Lane CJ. Application of the critical power concept to young swimmers. Pediatr Exerc Sci 1995;7:281-93.

24. Denadai BS, Donega MR, Greco CC. Comparação entre a velocidade de limiar anaeróbio e a velocidade crítica em nadadores de 10 a 15 anos. Rev Paul Ed Fís 1997;1:128-33.

25. Denadai BS, Greco CC, Teixeira M. Blood lactate response and critical speed in swimmers aged 10-12 years of different standards. J Sports Sci 2000;18:779-84

26. Mader A, Liesen H, Heck H, Philippi H, Rost R, Scharch PA, et al. Zur beurteilung der sportartspezifischen ausdauerlei-stungsfahigkeit. Sportarzt Sportmed 1976;27:80-8.

27. Vanderwalle H, Kapitaniak, B, Grun, S, Raveneau, S, Monod, H. Comparison between a 30-s-all-out test and a time-work test on a cycle ergometer. Eur J Appl Physiol 1989;58:375-81.

28. Wakayoshi K, Ilkuta K, Yoshida T, Udo M, Moritani T, Mutoh Y, et al. Determination and validity of critical velocity as an index of swimming performance in the competitive swimmer. Eur J Appl Physiol 1992;64: 153-7.

29. Wakayoshi K, Yoshida T, Udo M, Harada T, Moritani T. Does critical swimming velocity represent exercise intensity at maximal lactate steadystate? Eur J Appl Physiol 1993;66:90-5.

30. Eriksson BO, Saltin B. Muscle metabolism during exercise in boys aged 11-16 years compared to adults. Acta Paediatr Belg 1974;28:257-65.

31. Berg A, Kim SS, Keul J. Skeletal muscle enzyme activities in healthy young subjects. Int J Sports Med 1986;7:236-9.

32. Wirth A, Trager E, Scheele K, Mayer D, Diehm K, Reischle K, et al. Cardiopulmonary adjustment and metabolic response to maximal and submaximal physical exercise of boys and girls at different stages of maturity. Eur J Appl Physiol Occup Physiol 1978;39:229-40.

33. Tanaka H, Shindo M. Running velocity at blood lactate threshold of boys aged 6-15 years compared with untrained and trained young males. Int J Sports Med 1985;6:90-4.

34. Maglischo EW. Nadando ainda mais rápido. 1ํㅗ ed. São Paulo: Manole, 1999.

35. Olbrecht J, Madsen O, Mader A, Liesen H, Hollmann W. Relationship between swimming velocity and lactic concentration during continuous and intermittent training exercises. Int J Sports Med 1985;6:74-7.

36. Dekerle J, Sidney M, Hespel JM, Pelayo P. Validity and reliability of critical speed, critical stroke rate, and anaerobic capacity in relation to front crawl swimming performances. Int J Sports Med 2002;23:93-8. 